Acta Crystallographica Section A

Foundations of Crystallography

ISSN 0108-7673

Received 5 March 2008

Accepted 21 April 2008

(C) 2008 International Union of Crystallography Printed in Singapore - all rights reserved

\section{Elastic properties of two-dimensional quasicrystals}

\author{
Hans Grimmer
}

\author{
Laboratory for Development and Methods, Condensed Matter Research with Neutrons and Muons, \\ Paul Scherrer Institut, CH-5232 Villigen PSI, Switzerland. Correspondence e-mail: \\ hans.grimmer@psi.ch
}

Quasicrystals (QC) with two-dimensional quasiperiodic and one-dimensional periodic structure are considered. Their symmetry can be described by embedding the three-dimensional physical space $V_{E}$ in a five-dimensional superspace $V$, which is the direct sum of $V_{E}$ and a two-dimensional internal space $V_{I}$. A displacement $\mathbf{v}$ in $V$ can be written as $\mathbf{v}=\mathbf{u}+\mathbf{w}$, where $\mathbf{u} \in V_{E}$ and $\mathbf{w} \in$ $V_{I}$. If the QC has a point group $P$ in $V_{E}$ that is crystallographic, it is assumed that $\mathbf{w}$ and a vector $\mathbf{u}^{\prime} \in V_{E}$ lying in the plane in which the crystal is quasiperiodic transform under equivalent representations of $P$, inequivalent ones if the point group is 5-, 8-, 10- or 12-gonal. From the Neumann principle follow restrictions on the form of the phonon, phason and phonon-phason coupling contributions to the elastic stiffness matrix that can be determined by combining the restrictions obtained for a set of elements generating the point group of interest. For the phonon part, the restrictions obtained for the generating elements do not depend on the system to which the point group belongs. This remains true for the phason and coupling parts in the case of crystallographic point groups but, in general, breaks down for the non-crystallographic ones. The form of the symmetric $12 \times 12$ matrix giving the phonon, phason and phonon-phason coupling contributions to the elastic stiffness is presented in graphic notation.

\section{Introduction}

In the following, so-called two-dimensional quasicrystals will be considered (Hu et al., 1996). They are periodic in one direction and quasiperiodic in the plane perpendicular to it. Five integers are needed to index their diffraction patterns.

The symmetry of a quasicrystal (QC) can be described by embedding physical space in an $n$-dimensional $(n \mathrm{D})$ superspace. In our case, the three-dimensional physical space $V_{E}$ is embedded in a five-dimensional space $V$, which is the direct sum of $V_{E}$ and a two-dimensional internal space $V_{I}$ (Bak, 1985; Hu et al., 2000).

A displacement $\mathbf{v}$ in $V$ can be written as $\mathbf{v}=\mathbf{u}+\mathbf{w}$, where $\mathbf{u} \in$ $V_{E}$ and $\mathbf{w} \in V_{I}$. The gradient of $\mathbf{u}$ describes the change in the shape and volume of the unit cell, the gradient of $\mathbf{w}$ describes local rearrangements of the unit cells. $\mathbf{u}$ is referred to as the phonon part of $\mathbf{v}, \mathbf{w}$ as the phason part. The elastic energy density $F$ consists of three terms:

$$
F=F^{u}+F^{w}+F^{u w}=\frac{1}{2} C_{i j k l} E_{i j} E_{k l}+\frac{1}{2} K_{i j k l} W_{i j} W_{k l}+R_{i j k l} E_{i j} W_{k l},
$$

where $E_{i j}$ is the phonon strain,

$$
E_{i j}=\frac{1}{2}\left(\partial u_{i} / \partial x_{j}+\partial u_{j} / \partial x_{i}\right), \quad i, j=1,2,3,
$$

and $W_{i j}$ is the phason strain,

$$
W_{i j}=\partial w_{i} / \partial x_{j}, \quad i=1,2, j=1,2,3 .
$$

Equation (1) shows that the energy density contributions $F^{u}$, $F^{w}$ and $F^{u w}$ are due to the phonon strain field $E_{i j}$, the phason strain field $W_{i j}$ and to phonon-phason coupling, respectively (Ding et al., 1993).

The point group $P$ in $V_{E}$ of a two-dimensional QC is either non-cubic crystallographic or 5-, 8-, 10- or 12-gonal (Hu et al., 1996). If $P$ contains an $N$-fold rotation or rotation-inversion axis with $N>2$, this axis will be in the direction in which the crystal is periodic. Conventionally, a Cartesian coordinate system is chosen with the third axis in this direction. According to the Neumann principle, the phonon, phason and coupling parts, $C_{i j k l}, K_{i j k l}$ and $R_{i j k l}$, of the elastic stiffness must be invariant under the elements of the point group $P$. Because $F$ is invariant under orthogonal transformations, the transformation behaviour of $C, K$ and $R$ is determined by the transformation behaviour of $E_{i j}$ and $W_{i j}$. These, in turn, are given by the transformation behaviour of $\mathbf{u}, \mathbf{x}$ and $\mathbf{w}$ according to equations (2) and (3). Whereas $\mathbf{u}$ and $\mathbf{x}$ transform as vectors in $V_{E}$, it is assumed that $\mathbf{w}$ and a vector $\mathbf{u}^{\prime} \in V_{E}$ lying in the plane in which the crystal is quasiperiodic transform under equivalent representations of $P$ if $P$ is crystallographic, inequivalent ones if $P$ is 5-, 8-, 10- or 12-gonal (Hu et al., 1996). The elastic stiffness being invariant under the inversion $\overline{1}$, the restrictions on its form following from the Neumann principle 
Table 1

Laue classes, corresponding point groups, numbers of independent elastic constants of two-dimensional quasicrystals.

\begin{tabular}{|c|c|c|c|c|c|c|c|c|}
\hline \multirow[b]{2}{*}{ System } & \multirow[b]{2}{*}{$\begin{array}{l}\text { Orientation } \\
\text { of the rotation } \\
\text { group }\end{array}$} & \multicolumn{3}{|c|}{ Point groups in the Laue class } & \multicolumn{4}{|c|}{ Independent components } \\
\hline & & $\begin{array}{l}\text { Rotation group } \\
=\text { symbol of } \\
\text { Laue class }\end{array}$ & & $\begin{array}{l}\text { Centro- } \\
\text { symmetric } \\
\text { group }\end{array}$ & $n_{C}$ & $n_{K}$ & $n_{R}$ & Sum \\
\hline Triclinic & & 1 & & $\overline{1}$ & 21 & 21 & 36 & 78 \\
\hline Monoclinic $\left(\| x_{3}\right)$ & $2 \| x_{3}$ & 112 & $11 m$ & $112 / m$ & 13 & 13 & 20 & 46 \\
\hline \multirow[t]{2}{*}{ Monoclinic $\left(\perp x_{3}\right)$} & $2 \| x_{1}$ & 211 & $m 11$ & $2 / m 11$ & 13 & 12 & 18 & 43 \\
\hline & $2 \| x_{2}$ & 121 & $1 m 1$ & $12 / m 1$ & & & & \\
\hline Tetragonal & $4\left\|x_{3}, 2\right\| x_{1}$ & 422 & $4 m m,(\overline{4} m 2, \overline{4} 2 m)$ & $\underline{4} / \mathrm{mmm}$ & 6 & 5 & 5 & 16 \\
\hline \multirow[t]{3}{*}{ Trigonal } & $3 \| x_{3}$ & 3 & & $\overline{3}$ & 7 & 7 & 12 & 26 \\
\hline & $3\left\|x_{3}, 2\right\| x_{1}$ & 321 & $3 m 1$ & $\overline{3} m 1$ & 6 & 5 & 6 & 17 \\
\hline & $3\left\|x_{3}, 2\right\| x_{2}$ & 312 & $31 m$ & $\overline{3} 1 m$ & & & & \\
\hline \multirow[t]{2}{*}{ Hexagonal } & $6 \| x_{3}$ & 6 & $\overline{6}$ & $6 / m$ & 5 & 5 & 8 & 18 \\
\hline & $6\left\|x_{3}, 2\right\| x_{1}$ & 622 & $6 m m,(\overline{6} m 2, \overline{6} 2 m)$ & $6 / \mathrm{mmm}$ & 5 & 4 & 4 & 13 \\
\hline Pentagonal & $5 \| x_{3}$ & 5 & & $\overline{5}$ & 5 & 5 & 6 & 16 \\
\hline Octagonal & $8\left\|x_{3}, 2\right\| x_{1}$ & 822 & $8 m m,(\overline{8} m 2, \overline{8} 2 m)$ & $8 / \mathrm{mmm}$ & 5 & 4 & 1 & 10 \\
\hline \multirow[t]{2}{*}{ Dodecagonal } & $12 \| x_{3}$ & 12 & $\overline{12}$ & $12 / m$ & 5 & 5 & 0 & 10 \\
\hline & $12\left\|x_{3}, 2\right\| x_{1}$ & 1222 & $12 m m,(\overline{12} m 2, \overline{12} 2 m)$ & $12 / \mathrm{mmm}$ & 5 & 4 & 0 & 9 \\
\hline
\end{tabular}

will depend only on the Laue class of the point group. Therefore, the restrictions can be found by determining them for the pure rotation group in each Laue class.

Because $E_{i j}$ is symmetric in $i$ and $j$ (i.e. $E_{i j}=E_{j i}$ ) according to equation (2), $E_{i j}$ has only six independent components, which are conventionally arranged as

$$
\begin{gathered}
E_{1}=E_{11}, E_{2}=E_{22}, E_{3}=E_{33}, E_{4}=2 E_{23}, \\
E_{5}=2 E_{31}, E_{6}=2 E_{12} .
\end{gathered}
$$

$W_{i j}, i=1,2, j=1,2,3$, also has six independent components, which will be arranged in the order

$$
\begin{gathered}
W_{1}=W_{11}, W_{2}=W_{22}, W_{3}=W_{12}, W_{4}=W_{21}, \\
W_{5}=W_{13}, W_{6}=W_{23} .
\end{gathered}
$$

Equation (1) can then be written as

$$
F=\frac{1}{2} C_{\mu \nu} E_{\mu} E_{\nu}+\frac{1}{2} K_{\mu \nu} W_{\mu} W_{\nu}+R_{\mu \nu} E_{\mu} W_{\nu},
$$

where Greek indices run from 1 to 6 . The matrices $C_{\mu \nu}$ and $K_{\mu \nu}$ are chosen symmetric in $\mu$ and $v$ because an antisymmetric part does not contribute to $F$ according to (6).

Following $\mathrm{Hu}$ et al. (2000), we define the phonon and phason parts of the elastic stress by

$$
T_{i j}=\partial F / \partial E_{i j} \quad \text { and } \quad H_{k l}=\partial F / \partial W_{k l} .
$$

Notice that $i, j, l=1,2,3$ whereas $k=1,2 ; T_{i j}$ is symmetric in $i$ and $j$. Inserting (1) into (7), we obtain the generalized Hooke law

$$
\begin{aligned}
T_{i j} & =C_{i j k l} E_{k l}+R_{i j k l} W_{k l}, \\
H_{i j} & =R_{k l i j} E_{k l}+K_{i j k l} W_{k l} .
\end{aligned}
$$

$$
T_{1}=T_{11}, T_{2}=T_{22}, T_{3}=T_{33}, T_{4}=T_{23}, T_{5}=T_{31}, T_{6}=T_{12}
$$

and

$H_{1}=H_{11}, H_{2}=H_{22}, H_{3}=H_{12}, H_{4}=H_{21}, H_{5}=H_{13}, H_{6}=H_{23}$,

Hooke's law can be written as

$$
\begin{gathered}
T_{\mu}=C_{\mu \nu} E_{v}+R_{\mu \nu} W_{v}, \\
H_{\mu}=R_{\nu \mu} E_{v}+K_{\mu \nu} W_{v},
\end{gathered}
$$

or, in matrix form,

$$
\left[\begin{array}{c}
T \\
H
\end{array}\right]=\left[\begin{array}{cc}
C & R \\
R^{T} & K
\end{array}\right]\left[\begin{array}{c}
E \\
W
\end{array}\right],
$$

where the $12 \times 12$ matrix is symmetric.

The form of the parts $C, R$ and $K$ of this matrix were first studied for icosahedral quasicrystals by Levine et al. (1985) and by Bak (1985). They found that the numbers of independent elastic constants determining $C, K$ and $R$ are $n_{C}=2$, $n_{K}=2$ and $n_{R}=1$, respectively. For the 5-, 8-, 10- and 12-gonal Laue classes, the form of $C, R$ and $K$ has been given by $\mathrm{Hu}$ et al. (2000) in their Table 4. Notice that they arranged the six phason components in a different order.

Earlier, Hu et al. (1996) had determined for each non-cubic crystallographic or 5-, 8-, 10- or 12-gonal Laue class the quadratic expressions in the Cartesian components $E_{i j}$, the quadratic expressions in $W_{i j}$, and the expressions linear in both $E_{i j}$ and $W_{i j}$ that are invariant under the operations of the rotation group contained in the Laue class. In principle, these invariants determine the restrictions on the form of $C, K$ and $R$, respectively. Unfortunately, the list of invariants given in

Defining 
Table 2 of Hu et al. (1996) contains a number of errors. For convenience, the forms of $C, K$ and $R$ should be given directly, as was done by Hu et al. (2000) for the 5-, 8-, 10- and 12-gonal Laue classes. In $\$ 2$, the analogous results will be presented for the non-cubic crystallographic Laue classes. Table 1 lists these and the 5-, 8-, 10- or 12-gonal Laue classes together with the numbers $n_{C}, n_{K}, n_{R}$, as determined by $\mathrm{Hu}$ et al. (1996). The monoclinic Laue class as well as the trigonal and pentagonal Laue classes with more than one symmetry direction are given in different orientations, expressed by the Hermann-Mauguin symbols of the corresponding point groups. The conventions underlying the Hermann-Mauguin symbol define the orientation of the point group, as recalled in the second column of Table 1. Notice that $n_{K}$ and $n_{R}$ depend on whether the monoclinic axis lies in the periodic direction or the quasiperiodic plane. The symbol of the rotation group will also be used to denote the corresponding Laue class.

\section{The elastic matrix for two-dimensional quasicrystals with crystallographic point group}

Consider as a simple example the Laue class containing the point group 211, i.e. the point group generated by a $180^{\circ}$ rotation with axis along $x_{1}$. This operation leaves the first component of a vector invariant and changes the sign of the second and third components. $\mathbf{u}$ and $\mathbf{x}$ transform as vectors in three-dimensional space and $\mathbf{w}$ as a vector in the $x_{1} x_{2}$ plane. For any stress value, the elastic energy density $F$ remains invariant under the point-group operations of the QC. It then follows from (1) that all stiffness components with an odd number of indices 1 will vanish because for these an odd number among the four indices is either 2 or 3 . The $12 \times 12$ matrix then has the form given in Fig. 1.

The notation of Nye (1985) is used in Fig. 1: points denote vanishing components and filled circles components whose

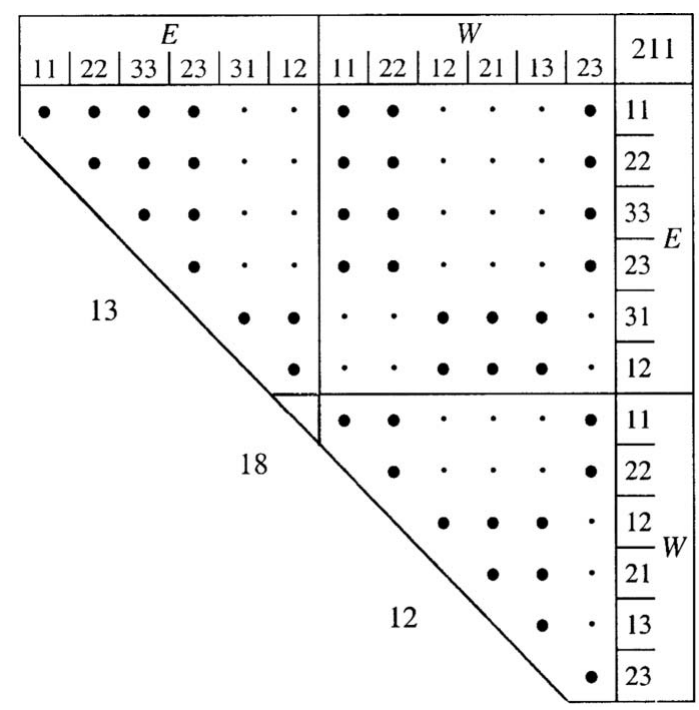

Figure 1

Form of the elastic stiffness matrix for two-dimensional quasicrystals in Laue class 211. values are not restricted by Neumann's law. Below the main diagonal, the numbers of independent components are given: $n_{C}=13, n_{R}=18, n_{K}=12$. The elastic stiffness matrix being symmetric, the value of each component below the main diagonal is equal to the value of the corresponding component above.

For Laue class 1, there are no restrictions, the corresponding Nye diagram contains only filled circles, $n_{C}=21, n_{R}=$ $36, n_{K}=21$.

Similarly to the Laue class 211, it follows from (1) that for 121 all stiffness components with an odd number of indices 2 will vanish, and that for 112 all stiffness components with an odd number of indices 3 will vanish, leading to the Nye diagrams given in Figs. 2 and 3.

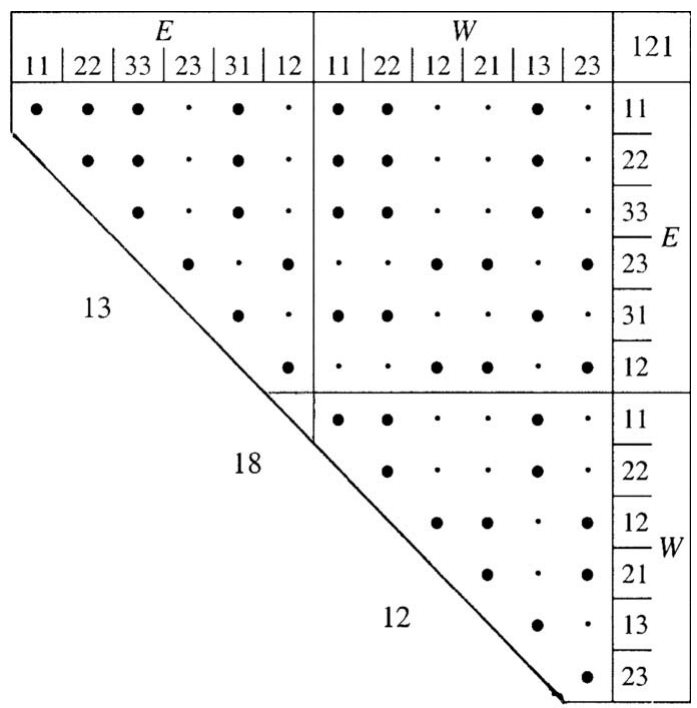

Figure 2

Form of the elastic stiffness matrix for two-dimensional quasicrystals in Laue class 121.

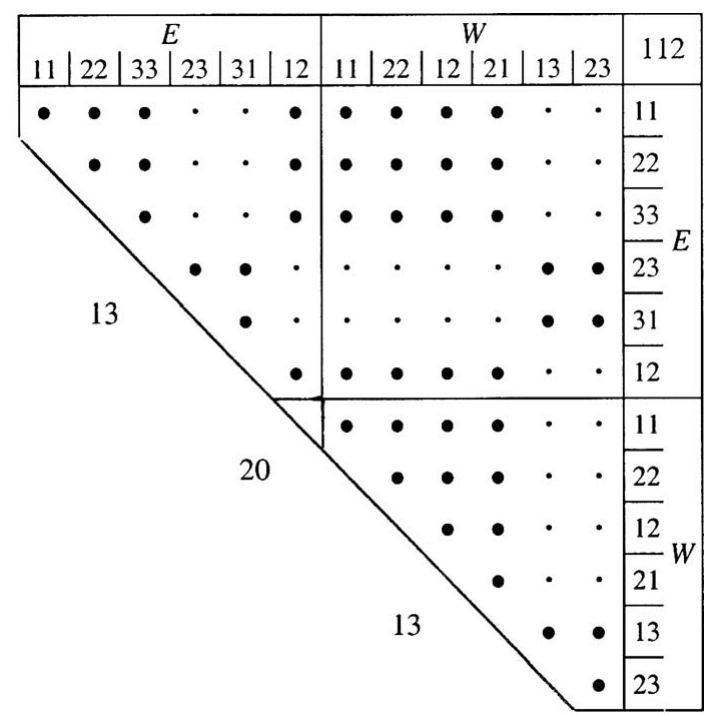

Figure 3

Form of the elastic stiffness matrix for two-dimensional quasicrystals in Laue class 112. 
Next we consider the Laue class containing the point group 222 , i.e. the point group generated by two $180^{\circ}$ rotations, with axes along $x_{1}$ and $x_{2}$, respectively. The matrix simultaneously satisfies the restrictions 211 and 121 (as well as 112). The result is shown in Fig. 4.

We now consider the Laue class containing the point group 4 , i.e. the point group generated by a $90^{\circ}$ rotation with axis along $x_{3}$. It maps the vector components $1 \rightarrow 2,2 \rightarrow-1,3 \rightarrow 3$, i.e. the components of $E$ and $W$ as follows

$$
\begin{array}{lllllll}
E_{i j} & 11 \rightarrow 22 & 22 \rightarrow 11 & 33 \rightarrow 33 & \begin{array}{r}
23 \rightarrow-13 \\
=-31
\end{array} & \begin{array}{c}
31 \rightarrow 32 \\
=23
\end{array} & \begin{array}{c}
12 \rightarrow-21 \\
=-12
\end{array} \\
E_{\mu} & 1 \rightarrow 2 & 2 \rightarrow 1 & 3 \rightarrow 3 & 4 \rightarrow-5 & 5 \rightarrow 4 & 6 \rightarrow-6 \\
W_{i j} & 11 \rightarrow 22 & 22 \rightarrow 11 & 12 \rightarrow-21 & 21 \rightarrow-12 & 13 \rightarrow 23 & 23 \rightarrow-13 \\
W_{\mu} & 1 \rightarrow 2 & 2 \rightarrow 1 & 3 \rightarrow-4 & 4 \rightarrow-3 & 5 \rightarrow 6 & 6 \rightarrow-5
\end{array}
$$

$R_{\mu \nu}$ then transforms as

$\begin{array}{rrrllllrrrrrr}11 & 12 & 13 & 14 & 15 & 16 & \rightarrow & 22 & 21 & -24 & -23 & 26 & -25 \\ 21 & 22 & 23 & 24 & 25 & 26 & & 12 & 11 & -14 & -13 & 16 & -15 \\ 31 & 32 & 33 & 34 & 35 & 36 & & 32 & 31 & -34 & -33 & 36 & -35 \\ 41 & 42 & 43 & 44 & 45 & 46 & & -52 & -51 & 54 & 53 & -56 & 55 \\ 51 & 52 & 53 & 54 & 55 & 56 & & 42 & 41 & -44 & -43 & 46 & -45 \\ 61 & 62 & 63 & 64 & 65 & 66 & -62 & -61 & 64 & 63 & -66 & 65\end{array}$

A similar procedure can be applied to $C$ and $K$. The ensuing necessary and sufficient restrictions for invariance of $R, C$ and $K$ are given in Fig. 5 .

The point group 422 is generated by a $90^{\circ}$ rotation about $x_{3}$ and a $180^{\circ}$ rotation about $x_{1}$. The matrix for Laue class 422 simultaneously satisfies the restrictions 4 and 211 . The result is shown in Fig. 5.

We now consider the Laue class containing point group 3, i.e. the point group generated by a $120^{\circ}$ rotation with axis along $x_{3}$. It maps the vector components $1 \rightarrow \mathrm{c} 1+\mathrm{s} 2,2 \rightarrow$ $-\mathrm{s} 1+\mathrm{c} 2,3 \rightarrow 3$, where $\mathrm{c}=\cos 120^{\circ}=-\frac{1}{2}, \mathrm{~s}=\sin 120^{\circ}=\frac{1}{2} \sqrt{ } 3$. The result is given in Fig. 6.

The point group 321 is generated by a $120^{\circ}$ rotation about $x_{3}$ and a $180^{\circ}$ rotation about $x_{1}$. The matrix for Laue class 321 simultaneously satisfies the restrictions for 3 and for 211.

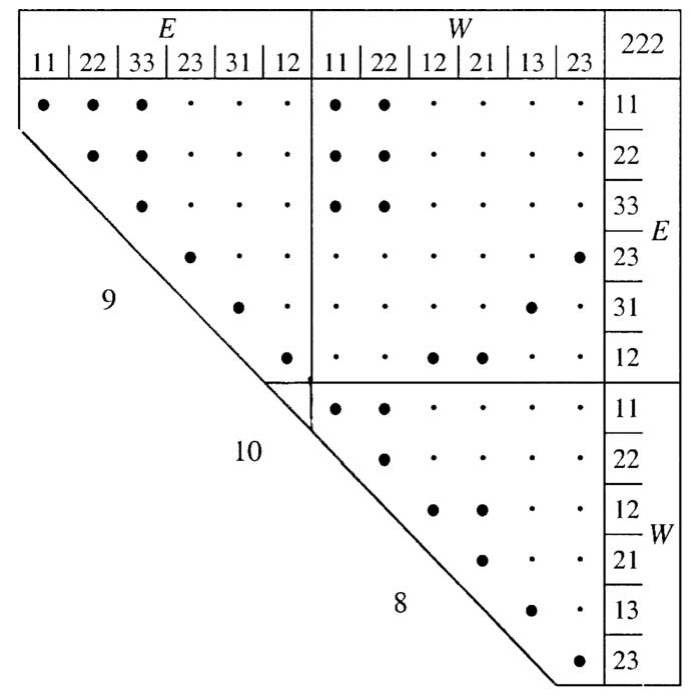

Figure 4

Form of the elastic stiffness matrix for two-dimensional quasicrystals in Laue class 222 .
Similarly, the matrix for Laue class 312 satisfies the restrictions for 3 and 121. The matrix for Laue class 6 satisfies the restrictions for 3 and 112; the matrix for Laue class 622 satisfies the restrictions for 6 and 211. All these result are shown in Fig. 6. Notice that the form of the matrix for Laue class 6 is a special case of the form for Laue class 4; an analogous result holds for 622 and 422 .

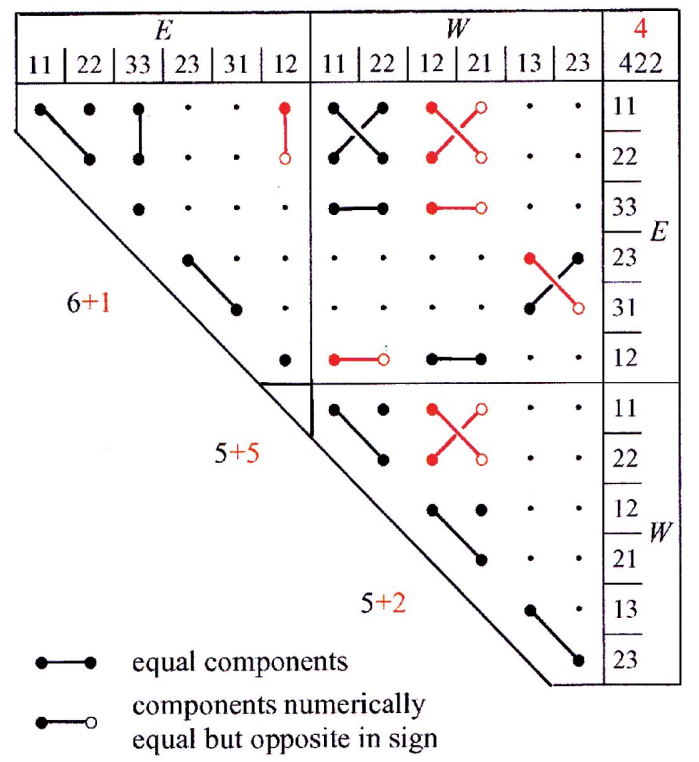

Figure 5

Form of the elastic stiffness matrix for two-dimensional quasicrystals in the Laue classes 4 and 422 . The black components are allowed in Laue class 422 , the black and red components in Laue class 4 .

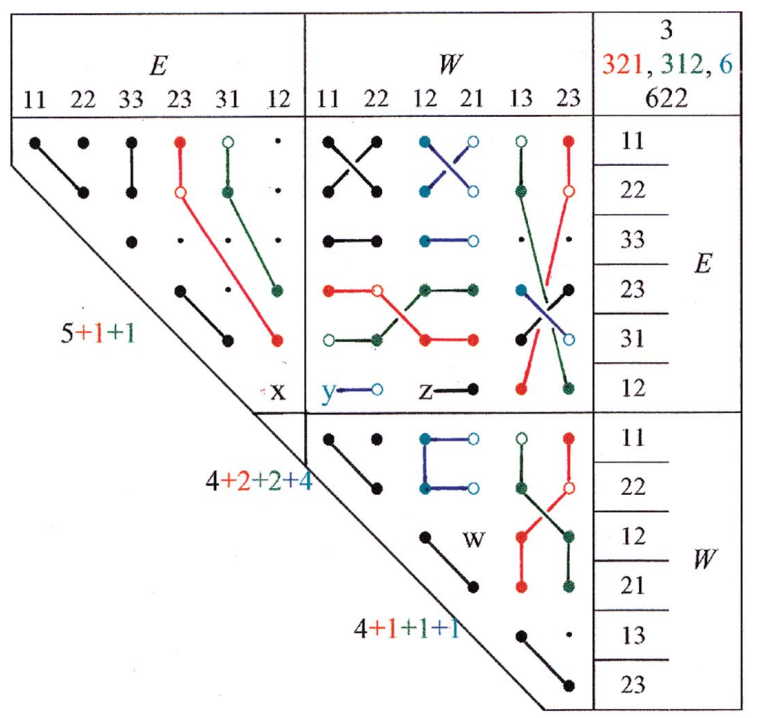

Figure 6

Form of the elastic stiffness matrix for two-dimensional quasicrystals in the trigonal and hexagonal Laue classes. $x: C_{66}=\frac{1}{2}\left(C_{11}-C_{12}\right) ; y: R_{61}=$ $-R_{62}=-\frac{1}{2}\left(R_{13}+R_{14}\right) ; z: R_{63}=R_{64}=\frac{1}{2}\left(R_{11}-R_{12}\right) ; w: K_{34}=K_{11}-K_{33}-$ $K_{12}$. The black components are allowed in Laue class 622 , the black and blue ones in 6 , the black and red ones in 321, the black and green ones in 312 , the black, blue, red and green ones in 3 . 


\section{The elastic matrix for two-dimensional quasicrystals with non-crystallographic point group}

\subsection{General considerations}

Under a rotation about the $z$ axis by an angle $2 \pi / N$, the phonon part $\mathbf{u}$ of a displacement $\mathbf{v}$ transforms with the transformation matrix $U$ and the phason part $\mathbf{w}$ with a transformation matrix $V$,

$$
\begin{aligned}
& U=\left(\begin{array}{ccc}
\cos 2 \pi / N & -\sin 2 \pi / N & 0 \\
\sin 2 \pi / N & \cos 2 \pi / N & 0 \\
0 & 0 & 1
\end{array}\right), \\
& V=\left(\begin{array}{cc}
\cos 2 k \pi / N & -\sin 2 k \pi / N \\
\sin 2 k \pi / N & \cos 2 k \pi / N
\end{array}\right)
\end{aligned}
$$

where $k=1$ in the crystallographic cases, for which $N=2,3,4$ or 6. Assuming $k=3$ for $N=5,8$ or 10 and $k=5$ for $N=12$, the results presented in Table 4(b) of Hu et al. (2000) are obtained. These values of $k$ are compatible with the perpendicular representations given in Table 1.10.5.1 of Janssen (2003) for

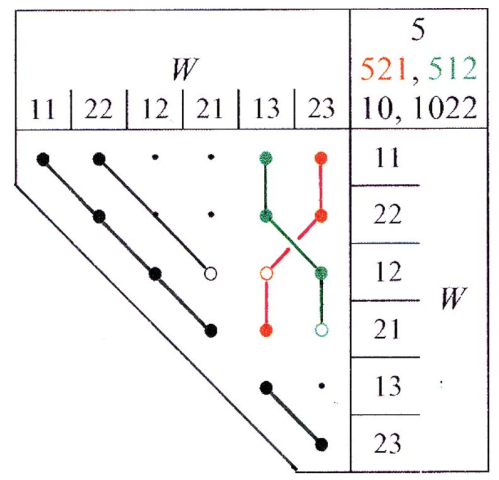

\section{Figure 7}

Form of the phason part $K$ of the elastic stiffness matrix for twodimensional quasicrystals in the pentagonal and decagonal Laue classes. The black components are allowed in Laue classes 10 and 1022, the black and red components in Laue class 521, the black and green components in Laue class 512, the black, red and green components in Laue class 5 . The number $n_{K}$ of independent components is 3 for Laue classes 10 and 1022, 4 for Laue classes 521 and 512, 5 for Laue class 5 .

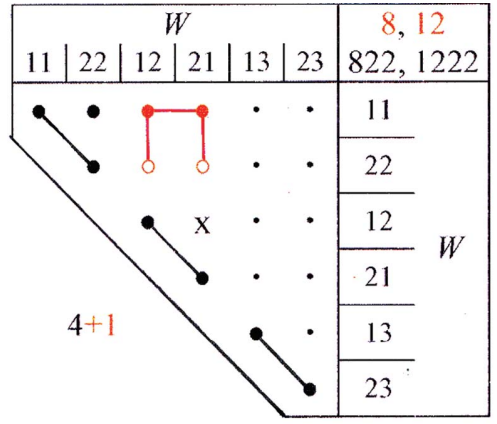

\section{Figure 8}

Form of the phason part $K$ of the elastic stiffness matrix for twodimensional quasicrystals in the octagonal and dodecagonal Laue classes. The black components are allowed in Laue classes 822 and 1222, the black and red components in Laue classes 8 and 12. $x: K_{34}=-K_{11}+K_{33}-$ $K_{12}$. the 5-, 8-, 10- and 12-gonal point groups containing only rotations, and they guarantee that the sum of the traces of the two matrices, $\operatorname{Tr}(U)+\operatorname{Tr}(V)$, is an integer.

Notice that $V$ differs for a rotation about the $z$ axis by an angle $\pi / 2$ according to whether the QC has octagonal or dodecagonal symmetry because the angle appearing in the trigonometric functions is $3 \pi / 2 \sim-\pi / 2$ in the first case and $5 \pi / 2 \sim \pi / 2$ in the second, in which $V$ is the same as for tetragonal symmetry. Also, the angle appearing for dodecagonal symmetry in the trigonometric functions of $V$ is $5 \pi / 3 \sim$ $-\pi / 3$ for a rotation by $\pi / 3$ and $10 \pi / 3 \sim-2 \pi / 3$ for a rotation by $2 \pi / 3$, i.e. it has opposite sign compared with hexagonal or trigonal symmetry. For rotations by $\pi$ about the $z$ axis, $V$ does not depend on the point group because the angle $\pi \sim-\pi$. Owing to the sign differences in the angles, we cannot expect in every case that the forms of the phason matrix $K$ and of the coupling matrix $R$ are for 8 special cases of the forms for 4 , and for 12 special cases of the forms for 6 (and 3). However, the forms for 8,10 and 12 must be special cases of the forms for 112, the forms for 12 even special cases of the forms for 4 .

All three parts, $C, K$ and $R$, of the elastic stiffness matrix simultaneously satisfy for 521 the restrictions for 5 and 211, for 512 the restrictions for 5 and 121, for 822 the restrictions for 8 and 211, for 1022 the restrictions for 10 and 211, and for 1222 the restrictions for 12 and 211.

\subsection{The phason part $K$}

Let me indicate how it can be shown that the component in red in Fig. 7 has the form given there. Assume that only one of the independent components is different from 0 and that $K_{1123}=d$. Fig. 7 then claims $K_{1123}=K_{2223}=-K_{1213}=K_{2113}=d$ and, because $K$ is symmetric, $K_{2311}=K_{2322}=-K_{1312}=K_{1321}=$ $d$. If we introduce the abbreviations $c=\cos 2 \pi / 5, s=\sin 2 \pi / 5$, $C=\cos 6 \pi / 5, S=\sin 6 \pi / 5$, a rotation by $2 \pi / 5$ about the $z$ axis will give for $K_{1123}^{\prime}$ according to equation (13):

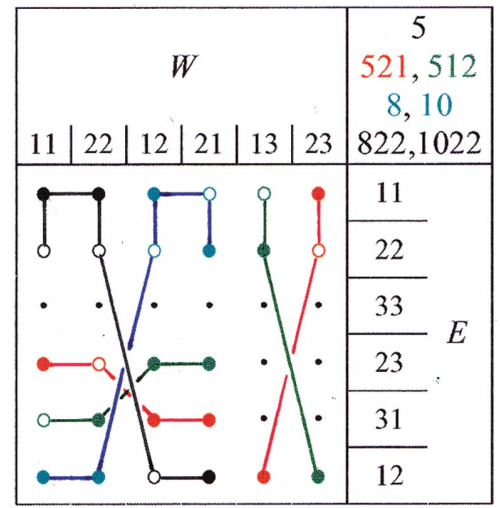

Figure 9

Form of the coupling part $R$ of the elastic stiffness matrix for twodimensional quasicrystals. The black component is allowed in Laue classes 822 and 1022, the black and blue components are allowed in Laue classes 8 and 10, the black and red components are allowed in Laue class 521, the black and green components in Laue class 512, the black, red, green and blue components in Laue class 5. The number $n_{R}$ of independent components is 1 for Laue classes 822 and 1022, 2 for Laue classes 8 and 10, 3 for Laue classes 521 and 512, 6 for Laue class 5 . 


$$
K_{1123}^{\prime}=d\left(C^{2} c+2 S C s-S^{2} c\right)=d .
$$

Notice that there are contributions only from the four terms where the index 3 appears in the fourth position. Similarly, one finds $K_{2223}^{\prime}=-K_{1213}^{\prime}=K_{2113}^{\prime}=d$, i.e. invariance under the rotation $2 \pi / 5$, which generates the point group 5 .

Figs. 8 and 5 show that the form for Laue classes 8 and 12 is a special case of the form for 4 , the form for 822 and 1222 a special case of the form for 422; in the cases of 12 and 1222 , this had to be expected, as shown in $\$ 3.1$. Notice that, although 3 is an oriented subgroup of 12 and 1222, the forms for 12 and 1222 are not special cases of the form for 3 .

Notice that the third and fourth entries in the last line in Table $4(d)$ of Hu et al. (2000) are interchanged, destroying the symmetry of the matrix $K$.

\subsection{The coupling part $R$}

Although 4 is an oriented subgroup of 8 and 822 , the forms of $R$ for 8 and 822, shown in Fig. 9, are not special cases of the form for 4 . The coupling part $R$ vanishes for the Laue classes 12 and 1222. It follows that the requirement that they be special cases of the forms for 4 and 422, respectively, is satisfied in a trivial manner.

\section{Discussion}

According to $\mathrm{Hu}$ et al. (1996), it has been assumed in all cases with crystallographic point group that $\mathbf{w}$ and a vector $\mathbf{u} \in V_{E}$ lying in the plane in which the crystal is quasiperiodic transform under equivalent representations. The restrictions on the form of the matrix following e.g. from invariance under a $180^{\circ}$ rotation then depend only on the axis of the rotation, not on the point group of the QC.

The phonon matrix $C$ does not depend on the transformation properties of $\mathbf{w}$. It has the same form for the Laue classes 5, 8, 10 and 12 as for the Laue class 6, according to the theorem of Hermann (1934) which states that for all tensors of rank $n$ a symmetry rotation of order $>n$ leads to the same restrictions as a symmetry rotation of infinite order. The matrix $C$ has the same form for 6 and 622 and therefore also for 521, 512, 822, 1022 and 1222.

If the two-dimensional QC has a 5-, 8-, 10- or 12-gonal point group, w will transform according to the matrix $V$ with $k \neq 1$ [see equation (13)], not as the component of $\mathbf{u}$ in the $x y$ plane. Once the forms of $K$ and $R$ have been determined for the point groups $5,8,10$ and 12 , they can be obtained as follows for the remaining 5-, 8-, 10- or 12-gonal Laue classes. For 521, the phason matrix $K$ simultaneously satisfies the restrictions following from the point-group symmetry $5 \| z$ and $2 \| x$, i.e. $K[521]=K[5] \cap K[211] ; K[211]$ is given in the lower part of our Fig. 1. Similarly, $K[512]=K[5] \cap K[121], K[N 22]=K[N] \cap$ $K[211]$, where $N=8,10,12$ and where $K[121]$ is the matrix given in the lower part of our Fig. 2. Analogous results hold for the phonon-phason mixing matrices $R: R[521]=R[5] \cap$ $R[211], R[512]=R[5] \cap R[121], R[N 22]=R[N] \cap R[211]$, where $R[211]$ and $R[121]$ are the matrices given in the upper right parts of our Figs. 1 and 2, respectively.

From Figs. 7-9, it follows that $K[5] \neq K[10] \neq K[8]=K[12]$ and $R[5] \neq R[10]=R[8] \neq R[12]$, showing that the theorem of Hermann (1934) holds only for the phonon part, where $C[5]=$ $C[10]=C[8]=C[12]=C[6]$.

A similar situation holds for the matrices describing the piezoelectric effect, as shown by the results of $\mathrm{Hu}$ et al. (1997), where the phason part $d^{(2)}$ of the piezoelectric matrix has the same form for point groups 4 and 6 as well as for point groups $\overline{8}$ and $\overline{12}$. However, the form for $\overline{8}$ is not the same as, or a special case of, the form for 4 although 4 is a subgroup of $\overline{8}$ and 6 a subgroup of $\overline{12}$.

The author wishes to thank T. Janssen for helpful discussions.

\section{References}

Bak, P. (1985). Phys. Rev. B, 32, 5764-5772.

Ding, D. H., Yang, W. G., Hu, C. Z. \& Wang, R. H. (1993). Phys. Rev. $B, \mathbf{4 8}, 7003-7010$.

Hermann, C. (1934). Z. Kristallogr. 89, 32-48.

Hu, C. Z., Wang, R. H. \& Ding, D. H. (2000). Rep. Prog. Phys. 63, 1-39.

Hu, C. Z., Wang, R. H., Ding, D. H. \& Yang, W. G. (1997). Phys. Rev. $B, \mathbf{5 6}, 2463-2468$.

Hu, C., Wang, R., Yang, W. \& Ding, D. (1996). Acta Cryst. A52, 251-256.

Janssen, T. (2003). International Tables for Crystallography, Vol. D, edited by A. Authier, pp. 243-264. Dordrecht: Kluwer Academic Publishers.

Levine, D., Lubensky, T. C., Ostlund, S., Ramaswamy, S., Steinhardt, P. J. \& Toner, J. (1985). Phys. Rev. Lett. 54, 1520-1523.

Nye, J. F. (1985). Physical Properties of Crystals: their Representation by Tensors and Matrices. Oxford: Clarendon Press. 